\title{
Automated and Continual Determination of Radio Telescope Reference Points With Sub-mm Accuracy Results from a campaign at the Onsala Space Observatory
}

\author{
Michael Lösler • Rüdiger Haas . \\ Cornelia Eschelbach
}

Received: date / Accepted: date

\begin{abstract}
The Global Geodetic Observing System (GGOS) requires sub-mm accuracy, automated and continual determinations of the so-called local ties vectors at co-location stations. Co-location stations host instrumentation for several space geodetic techniques and the local tie surveys involve the relative geometry of the reference points of these instruments. Thus, these reference points need to be determined in a common coordinate system, which is a particular challenge for rotating equipment like radio telescopes for geodetic Very Long Baseline Interferometry. In this work we describe a concept to achieve automated and continual determinations of radio telescope reference points
\end{abstract}

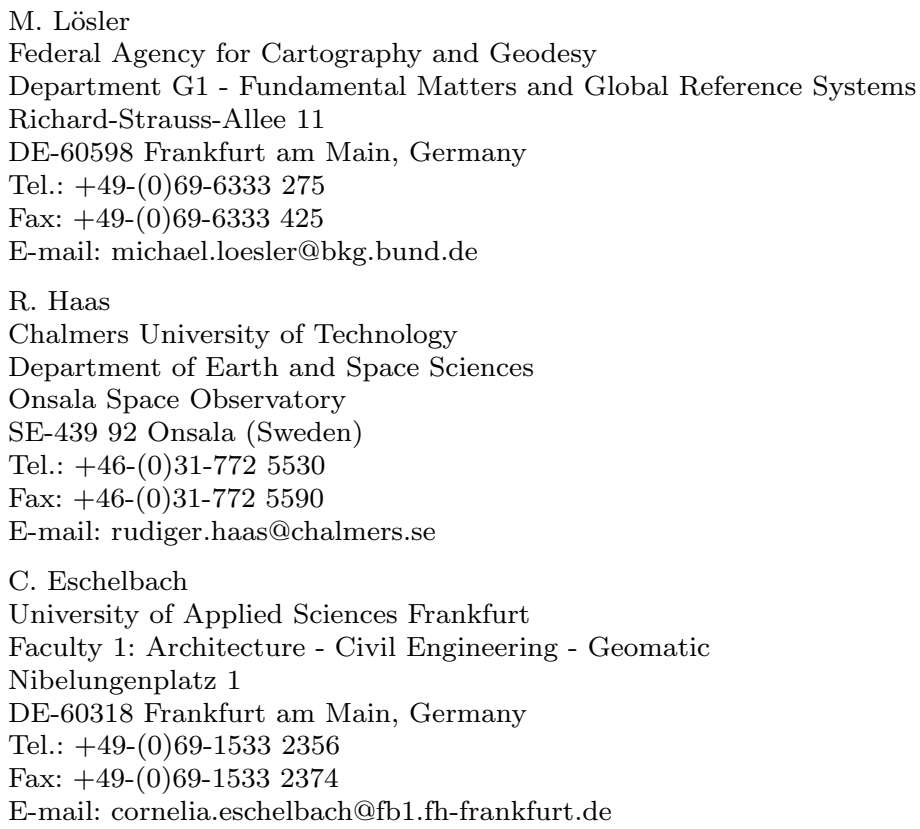


with sub-mm accuracy. We developed a monitoring system, including Javabased sensor communication for automated surveys, network adjustment, and further data analysis. This monitoring system was tested during a monitoring campaign performed at the Onsala Space Observatory in the summer of 2012. The results obtained in this campaign show that it is possible to perform automated determination of a radio telescope reference point during normal operations of the telescope. Accuracies on the sub-mm level can be achieved, and continual determinations can be realized by repeated determinations and recursive estimation methods.

Keywords VLBI · Radio Telescope · Reference Point Determination · Monitoring $\cdot$ Least Squares

\section{Introduction}

The globally distributed infrastructure of equipment for space geodetic measurements is of great importance for geodesy and geoscience research in general and for the Global Geodetic Observing System (GGOS) in particular. It forms the backbone of GGOS which is the 'flagship' of the International Association of Geodesy (IAG) and aims among other things at combining and integrating the various space geodetic measurements (Rummel et al., 2005; Plag and Pearlman, 2009). In order to achieve meaningful inter-technique combinations, GGOS makes use of co-location stations that host infrastructure for several space geodetic techniques. At these co-location stations the relative geometry of the technique specific reference points needs to be known with high accuracy, including potential variation with time (Ray and Altamimi, 2005). The individual techniques have both electronic and geometric reference points and the relation between those two has to be determined by the respective technique, which is out of scope for this work. The relative geometry between the geometric reference points of different techniques is usually referred to as local tie vectors, and requires to determine these reference points in a common geocentric coordinate system. To achieve the ambitious goals of GGOS, local tie surveys are required to be performed with sub-mm accuracy, fully automated, and almost continuously (Rothacher et al., 2009).

Traditionally, local tie surveys at co-location stations are done with classical geodetic survey techniques and with low repetition rate. This is mainly due to that the local tie surveys usually are difficult and time consuming engineering tasks of high complexity. The complexity lies for example in that in some cases the geometrical reference points cannot be observed directly, e.g. the reference point of VLBI radio telescopes or an optical telescope used for satellite laser ranging (SLR) is defined as the intersection between the primary axis and secondary axis. If these two axes do not intersect, the reference point is the normal projection of the secondary axis onto the primary axis. This position is geometrically invariant, i.e. independent of the pointing direction of the radio telescope see e.g. Eschelbach (2002). In these cases indirect survey methods need to be applied. Indirect methods for rotating instrumentation 
like telescopes for VLBI or SLR usually exploit the instrument's symmetry properties, see e.g. Sarti et al. (2004). For these methods the instrumentation needs to be positioned according to a predefined schedule in order to be able to do the survey observations. This in turn means an interruption of the normal operations of the instrument. An approach to achieve reference point determination of a rotating instrument also during normal operations has been proposed by Lösler (2009).

Even though sub-mm accuracies can be achieved and have been reported previously, e.g. Eschelbach (2002), Sarti et al. (2004), Dawson et al. (2007), Lösler and Haas (2009), the aspects of automation and continual measurements have been hardly addressed so far. With the advent of total stations being fully automated and programmable instruments, automated monitoring becomes a viable approach for the determination of reference points and for local tie surveys. Conventional monitoring is confined to observe semi-fixed discrete points. This approach can be adopted to check the stability of radio telescope monuments (e.g. Haas and Bergstrand (2010), Lösler et al. (2010)), and GNSS monuments (e.g. Haas et al. (2013)).

In this work we extend the monitoring approach to an automated and continual determination of radio telescope reference points. Section 2 briefly discusses radio telescope reference points and the difficulties involved in the determination, in particular the problems concerning the aspects of automation and continual measurements. This is followed by Section 3 that describes our concept to achieve automated and continual determinations of radio telescope reference points and the monitoring system that was developed for this purpose. This monitoring system is an improved and enhanced version of the HEIMDALL system described in Lösler et al. (2010).

Section 4 describes a monitoring campaign performed at the Onsala Space Observatory in the summer of 2012 applying this new monitoring system and the corresponding results that were obtained. Section 5 concludes this work with a short summary and conclusions.

\section{Determination of radio telescope reference points}

The reference point of rotating space geodetic instrumentation like radio telescopes for geodetic VLBI observations or optical telescopes for SLR measurements usually is defined as the intersection of the axes that the instrument is turning around. For example, the International VLBI Service for Geodesy and Astrometry (IVS) defines the reference point of azimuth-elevation type VLBI radio telescopes as the intersection between the azimuth and elevation axis (Nothnagel, 2009). In case these two axes do not intersect, the reference point is defined as the normal projection of the elevation axis onto the azimuth axis.

For most radio telescopes the reference point must be determined by indirect survey methods. Only for very few VLBI telescopes it is possible to survey this reference point directly. However, even in these special cases it is not possible to observe the reference point continually during normal operation of the 
telescope. Thus, also in these cases the aspect of continual measurements calls for indirect survey methods.

One way to indirectly derive the telescope reference point is to mount survey targets on the telescope structure and observe them with a total station that is placed on a survey pillar in the local survey network. Then the telescope is rotated in predefined steps so that the trajectory of each observed target lies on a circle-arc. Repeating this procedure in different telescope orientations with several targets makes it possible to estimate the reference point by 3D circle-fitting (e.g. Eschelbach (2002), Sarti et al. (2004), Dawson et al. (2007), Leinen et al. (2007)). As this method requires predefined telescope orientations, it is restricted in terms of automation and continual measurements.

Another approach for reference point determination is to apply the transformation model described by Lösler (2009). Through involving the telescope orientation angles, i.e. azimuth and elevation, as additional observations, this model describes a transformation from a turnable telescope frame to a local ground fixed reference system. This mathematical model also needs survey targets at the turnable part of the radio telescope, but in contrast to the $3 \mathrm{D}$ circle-fitting method it does not require predefined orientations. Comparisons between both, the circle-fitting method and the transformation model, yield highly consistent results (Lösler, 2008; Lösler and Haas, 2009). Since the transformation approach does not need predefined orientations, it can even be applied to surveys of telescopes during normal telescope operation, thus paving the way for automation and continual measurements. Simulation studies and first attempts of an automated monitoring suggest suitable results (e.g. Kallio and Poutanen (2012), Schmeing et al. (2010)).

The positions of the mounted targets are only semi-fixed in the telescope system. These targets and their orientations change as a function of the rotation sequence of the telescope and are sometimes not observable, e.g. due to blockage or misalignment between the survey target and the survey instrument. To increase the survey visibility, special survey targets can be used. A possible target type is the so-called $360^{\circ}$ prism. This kind of prism consists of a combination of several reflectors around a vertical axis. However, because of this construction, the $360^{\circ}$ prism causes systematic errors up to the centimeter-level, depending on the orientation of the prism with respect to the survey instrument (Favre and Hennes, 2000; Krickel, 2004). Other possibilities are so-called cateye reflectors with an opening angle of $\pm 60^{\circ}$, or active targets, that automatically detect and correct misalignments. These special targets are often used in metrology in combination with laser trackers. Generally, independent of the target type used, a full survey visibility is not possible, and the target incident angle has to be taken into account during the monitoring. Furthermore, to make a continual monitoring efficient, a common data flow starting at the observation level up to the reference point determination is required. 


\section{The concept of automated and continual determinations of a telescope reference point}

The automated and continual determination of the reference point of a radio telescope is a complex issue. The structure of this complex task becomes much clearer by defining subtasks as modules, solving them separately and using a standard communication interface for the communication between the modules. In the following we describe our approach to this problem and the monitoring system HEIMDALL that has been developed for this purpose. Figure 1 gives an overview of the monitoring system and the defined subtasks.

The first subtask is an observation plan for the survey system and is addressed by the observation plan module. Since the ultimate goal is to perform the survey during ongoing normal operation of the telescope, the observing plan should be derived based on a real VLBI schedule. During the actual survey this observation plan then controls all involved active sensors, like the total station, but also possible additional sensors like inclinometers. However, it is also plausible that the reference point determination is performed occasionally using a dedicated survey schedule.

The second subtask is addressed by the monitoring module that performs and records the actual measurements. Furthermore, the monitoring module also records non-time-critical environmental parameters like monument temperature, air temperature, pressure, humidity and telescope height variations. Most of these parameters affect the measurements and their influence must therefore be compensated either directly during the monitoring or in the later data analysis.

The remaining subtasks concern the data analysis, i.e. the network analysis, the determination of the reference point, and the combination of results, and are addressed by three individual modules. First, the observed network has to be determined by a least squares adjustment. The results of this adjustment are the estimated coordinates of the points at the telescope structure and their related variance-covariance-matrix. Second, these coordinates and the variance-covariance-matrix are used to estimate the reference point and additional telescope parameters. Finally, if the data of more than one epoch is available, recursive adjustment approaches can be applied to combine the corresponding results and methods for time series analysis can be performed.

Data management and data exchange play an important role for the whole monitoring system. An efficient and self-consistent management of large data sets is a basic requirement. Moreover, data records must be selectable, sortable, searchable, combinable etc. without an appreciable delay. These requirements can be fulfilled using a SQL database management system. Thus, we implemented the embedded database HSQLDB in the monitoring system to manage the complete data flow between the different modules. 


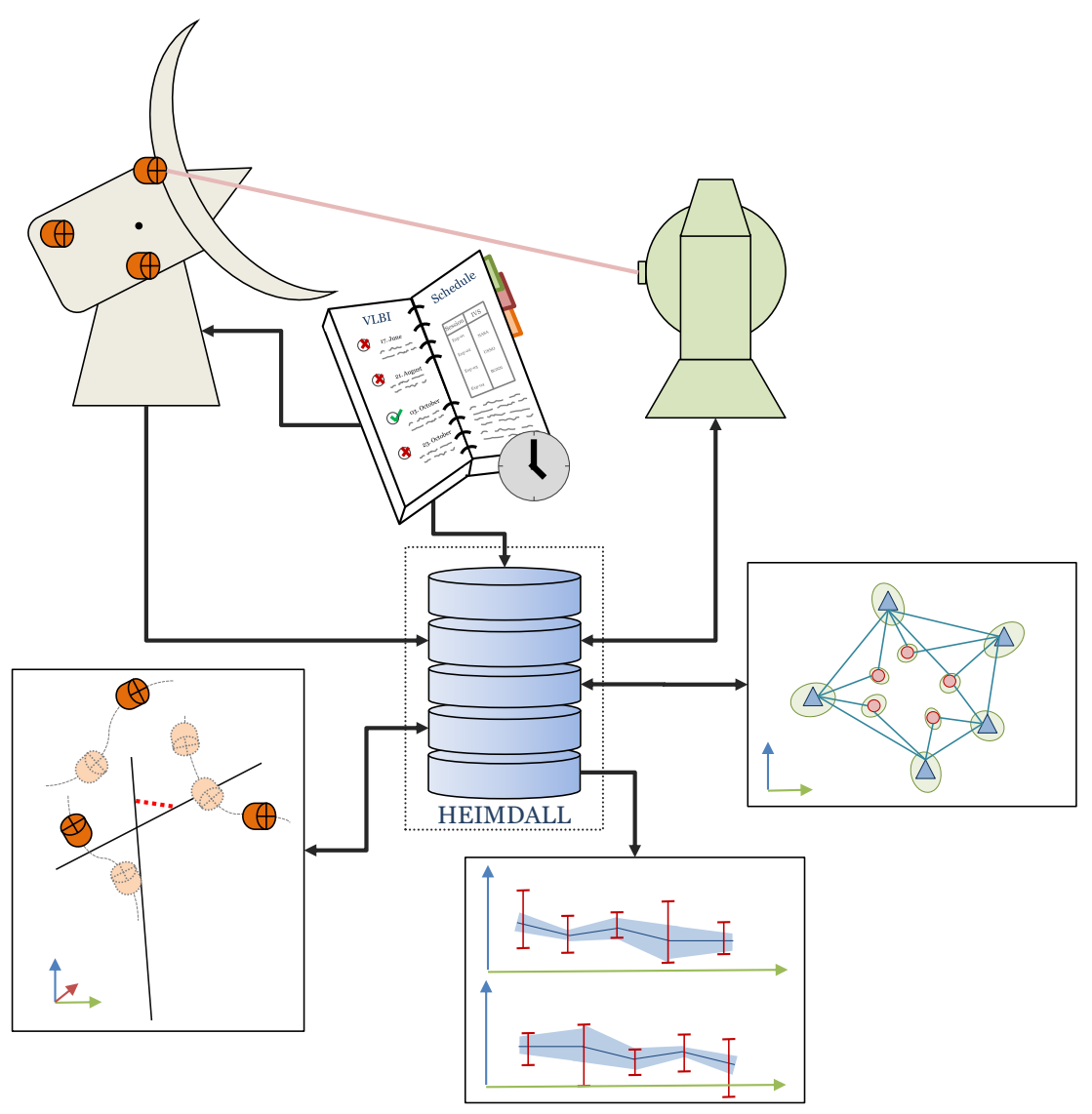

Fig. 1 Schematic representation of the monitoring system HEIMDALL for automated and continual determination of a telescope reference point.

\subsection{Observation plan module}

An observation plan has to be generated based on a telescope schedule, that could be either a VLBI schedule or a dedicated survey schedule. In both cases the schedule defines the telescope orientation with time. The observation plan needs to follow this schedule and to connect the survey stand points $\mathbf{P}_{\text {TS }}^{j}$ and the related surveyable targets at the radio telescope. To derive this plan an appropriate approximation of the reference point $\mathbf{P}_{\mathrm{RP}}^{0}$ and the coordinates $\mathbf{P}_{\text {Obs }}^{i}$ together with their normal directions $\mathbf{n}_{\text {Obs }}^{i}$ of several targets at the rotatable telescope structure must be available. Thus, an initial survey needs to be performed to determine the necessary a priori information.

To carry out observations between a total station and a radio telescope, two coordinate systems have to be combined. The first system is a local Cartesian ground fixed reference frame of the terrestrial surveying instrument. In general, it is part of the local site network and is realized by survey pillars. The second 
coordinate system is defined by the structure of an idealized radio telescope. The majority of the radio telescopes currently used in the IVS network is of azimuth-elevation type. Thus, we concentrate in the following on this telescope type. However, the described model and equations can be easily modified for any other type of radio telescope, too. The origin is given by the reference point $\mathbf{P}_{\mathrm{RP}}$ and the $\mathrm{x}$-axis and $\mathrm{z}$-axis correspond to the elevation- and azimuth axes, respectively. The $\mathrm{y}$-axis is perpendicular to the $\mathrm{x}$ - and z-axes. If both, the ground fixed reference system and the telescope system, are roughly congruent to each other when $\alpha=\epsilon=0^{\circ}$, the $\mathbf{P}_{\text {Obs }}^{i}(\alpha, \epsilon)$ can be formulated as function of the given telescope orientation

$$
\mathbf{P}_{\mathrm{Obs}}^{i}(\alpha, \epsilon)=\mathbf{P}_{\mathrm{RP}}^{0}+\mathbf{R}_{\alpha, \epsilon}\left(\mathbf{P}_{\mathrm{Obs}}^{i}-\mathbf{P}_{\mathrm{RP}}^{0}\right)
$$

with

$$
\mathbf{R}_{\alpha, \epsilon}=\mathbf{R}_{\alpha}^{z} \mathbf{R}_{\epsilon}^{x}
$$

where $\mathbf{R}$ denotes a rotation matrix. Note that in contrast to the elevation angle $\epsilon$ the azimuth angle $\alpha$ rotates clockwise. One way to derive the a priori $\mathbf{P}_{\mathrm{RP}}^{0}$ with sufficient accuracy is to use $\mathbf{P}_{\mathrm{L}}$ and $\mathbf{P}_{\mathrm{R}}$, the left and right end points of the elevation axis for one telescope position, respectively.

$$
\mathbf{P}_{\mathrm{RP}}^{0}=0.5\left(\mathbf{P}_{\mathrm{L}}+\mathbf{P}_{\mathrm{R}}\right)
$$

In general, the two coordinate systems differ in an azimuth orientation $O_{\alpha}$ with

$$
O_{\alpha} \approx \arctan \frac{y_{\mathbf{P}_{\mathbf{R}}}-y_{\mathbf{P}_{\mathrm{RP}}}^{0}}{x_{\mathbf{P}_{\mathbf{R}}}-x_{\mathbf{P}_{\mathrm{RP}}}^{0}}+\alpha_{\mathbf{P}_{\mathrm{R}}}
$$

where $\alpha_{\mathbf{P}_{\mathbf{R}}}$ is the azimuth angle that was used during the initial measurement of $\mathbf{P}_{\mathbf{R}}$. Equation 4 is an approximation formula because the azimuth axis is usually not parallel to the vertical axis of the local reference frame.

In fact, a complete observation of the mounted survey targets at $\alpha=\epsilon=0^{\circ}$ is usually not possible and the initial telescope angles $\alpha_{0}^{i}$ and $\epsilon_{0}^{i}$ of the i-th point $\mathbf{P}_{\text {Obs }}^{i}$ have to be taken into account. The modified rotation matrix $\mathbf{R}_{\alpha, \epsilon}$ is given by

$$
\mathbf{R}_{\alpha, \epsilon}=\mathbf{R}_{\alpha-O_{\alpha}}^{z} \mathbf{R}_{\epsilon-\epsilon_{o}^{i}}^{x} \mathbf{R}_{O_{\alpha}-\alpha_{o}^{i}}^{z}
$$

The normal vectors $\mathbf{n}_{\text {Obs }}^{i}$ of the mounted survey targets can be determined during the initial survey by measuring additionally to a second survey target that is centered in front of the actual survey target, while the symmetric axes of the two prisms are congruent. In case of glass prims the glass surface of the two prisms must be parallel to each other. Figure 2 illustrated a simple way to achieve the determination of the normal vector. A small RFI (reflector for fixed installations) is rigidly attached to a rotation symmetric plastic cylinder that is placed centrally in front of the survey prism so that the glass surfaces of 
the prism and the small RFI are parallel and the symmetric axes of the prism and the corner cube reflector are congruent. Measurements are conducted to both the small RFI and to the survey prism itself. The derived positions are then used to calculate the normal vector on the surface of the survey prism. Finally, the angle of incidence $\tau$ at the i-th target with respect to the survey point $\mathbf{P}_{\mathrm{TS}}$ is

$$
\cos \tau^{i}=\frac{\left[\mathbf{P}_{\mathrm{TS}}-\mathbf{P}_{\mathrm{Obs}}^{i}(\alpha, \epsilon)\right]^{T} \mathbf{R}_{\alpha, \epsilon} \mathbf{n}_{\mathrm{Obs}}^{i}}{\left|\mathbf{P}_{\mathrm{TS}}-\mathbf{P}_{\mathrm{Obs}}^{i}(\alpha, \epsilon)\right|\left|\mathbf{n}_{\mathrm{Obs}}^{i}\right|}
$$

This angle has to be compared with the specified opening angle that the manufacturer gives for the prism type in use, e.g. $\pm 45^{\circ}$, to check the accessibility. The planned survey points and their related target positions at the telescope are stored together with a timestamp in the observation plan table of the database. Moreover, observations to the local reference frame have to be defined to georeference the survey points with respect to each other and to proof the invariance of the instrument.

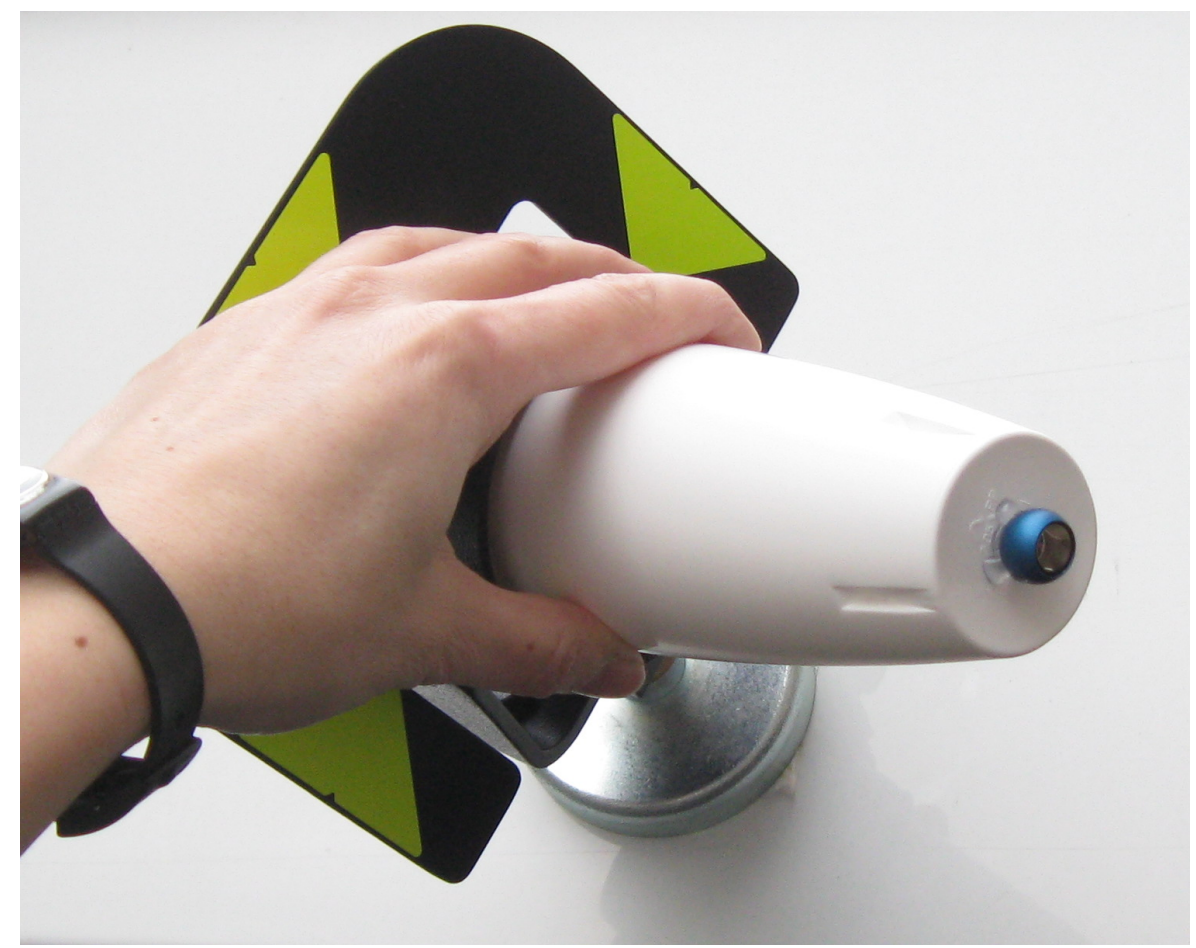

Fig. 2 A small RFI (reflector for fixed installations) is rigidly attached to a rotation symmetric plastic cylinder that is placed centrally in front of survey prism so that the RFI and the survey prism have parallel glass surfaces and congruent symmetry axes. Measurements to both the small RFI and the survey target prism allow then to determine the normal vector of the survey target prism. 


\subsection{Automated monitoring module}

The sensor communication and monitoring software was completely developed in the platform-independent language Java. The software can communicate with metrological devices and sensors that allow non-proprietary communication. Examples of such instrumentation are the total station of type Leica TS30 and the meteorological sensor MSR145 that both provide a non-proprietary application programming interface for direct communication over the standard serial ports RS-232. The meteorological parameters are used to correct the electronic distance measurements directly. Based on the observation plan, the total station locates the defined targets, starts the automatic target recognition (ATR), and - in case of success - the observations are saved together with the related recording time. Reference measurements to survey pillars of the local site network can be realized as often as needed, but at least at the beginning and in the end of the allocation of each survey point (cf. Burg (2012)).

Recordings of the angle encoder values of the radio telescope and further auxiliary data, e.g. height measurements performed with an invar rod, temperature measurements of the telescope structure, etc., are logged externally and continually. A correct assignment between these data and the geometric observations is carried out via synchronizing the recorded timestamps.

\subsection{Network adjustment module}

After finalizing the monitoring, the observed data is analyzed with a network adjustment module. The main tasks during the network adjustment are to connect the survey points and to determine realistic uncertainties. A spatial data analysis is necessary to derive the full variance-covariance-matrix $Q_{x y z}$ of the observed points. For this module the application Java Graticule 3D (JAG3D) is introduced to the analysis sequence (Lösler, 2012).

During a VLBI schedule, the telescope rotates continuously and no redundant measurements to the targets can be carried out. Thus, it is impossible to identify a blunder during the network adjustment. Blunders have therefore to be detected during the reference point estimation (cf. Lösler (2008)). However, the comparison between the a priori position and the a posteriori position of the targets gives a rough indication of an outlier. In general, the difference can be expected to be $<1 \mathrm{dm}$, so a threshold of about $1 \mathrm{~m}$ can be chosen to detect and exclude blunders. In principle, incorrect measurements result from observations to another target at the telescope and can be corrected (Mazura, 2012). On the other hand, dedicated observing plans give the possibility to observe the same targets several times, thus providing redundancy. This gives the opportunity to proof the uncertainty budget via variance-component-estimation (e.g. Crocetto et al. (2000), Koch (1999)).

Whereas the prisms on the survey pillars can be aligned to the instrument, the angle of incidence at the telescope targets is depending on the telescope 
orientation. Depending on the type of prisms used, the incidence angle causes lateral and radial deviations of the prism position. Corner cube reflectors with mirrors are not affected by an incorrect alignment, but glass prisms are.

For glass prisms the radial deviation $\varepsilon_{\text {radial }}$ from an inaccurate alignment can be assessed by (Pauli, 1969)

$$
\varepsilon_{\text {radial }}=d\left(n-\sqrt{n^{2}-\sin ^{2} \delta}\right)-e(1-\cos \delta)
$$

where $e$ is the distance between the front surface of the prism and the centersymmetric point, and $d$ is the distance between the front surface of the prism and the corner point of the triple prism. The ratio of the group refractive indices of glass and air are denoted by $n$, and $\delta$ is the angle of incidence. Furthermore, the lateral deviation $\varepsilon_{\text {lateral }}$ is (Rüeger, 1990)

$$
\varepsilon_{\text {lateral }}=(d-e) \sin \delta-d \sec \delta_{G} \sin \left(\delta-\delta_{G}\right)
$$

where $\delta_{G}=\arcsin \frac{\sin \delta}{n}$.

Figure 3 depicts the lateral and radial deviations depending on the angle of incidence $\delta$ for two glass prisms of type Leica GPR121 and Leica GMP104. For example, for a prism Leica GPR121 a misalignment of $\delta=35$ gon, leads to deviations of $\varepsilon_{\text {radial }} \approx 0.2 \mathrm{~mm}$ and $\varepsilon_{\text {lateral }} \approx 1.5 \mathrm{~mm}$ that need be taken into account in a deterministic way to avoid systematic errors.

Figure 4 describes the deterministic correction for spatial polar observations schematically. The true position of the survey target is denoted by $\mathbf{P}_{\mathrm{Obs}}^{\prime}$, but due to the misalignment between the the survey target and the survey instrument the recorded measurements refer to point $\mathbf{P}_{\text {Obs }}$. Thus, the recorded distances, vertical and horizontal angles need to be corrected. The radial and lateral deviations due to the misalignment can be calculated with equations 7

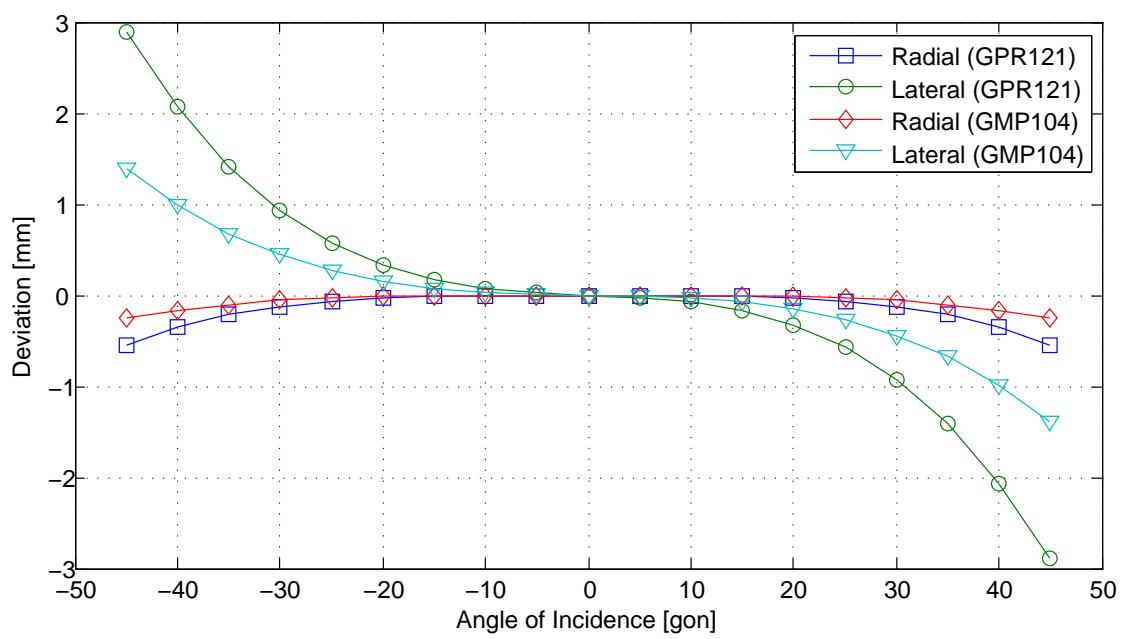

Fig. 3 Lateral and radial deviation for prisms of type GPR121 ( $d=39.8 \mathrm{~mm}, e=26.2 \mathrm{~mm})$ and GMP104 $(d=19.0 \mathrm{~mm}, e=12.52 \mathrm{~mm})$ with $n=1.53(\lambda=658 \mathrm{~nm})$. 


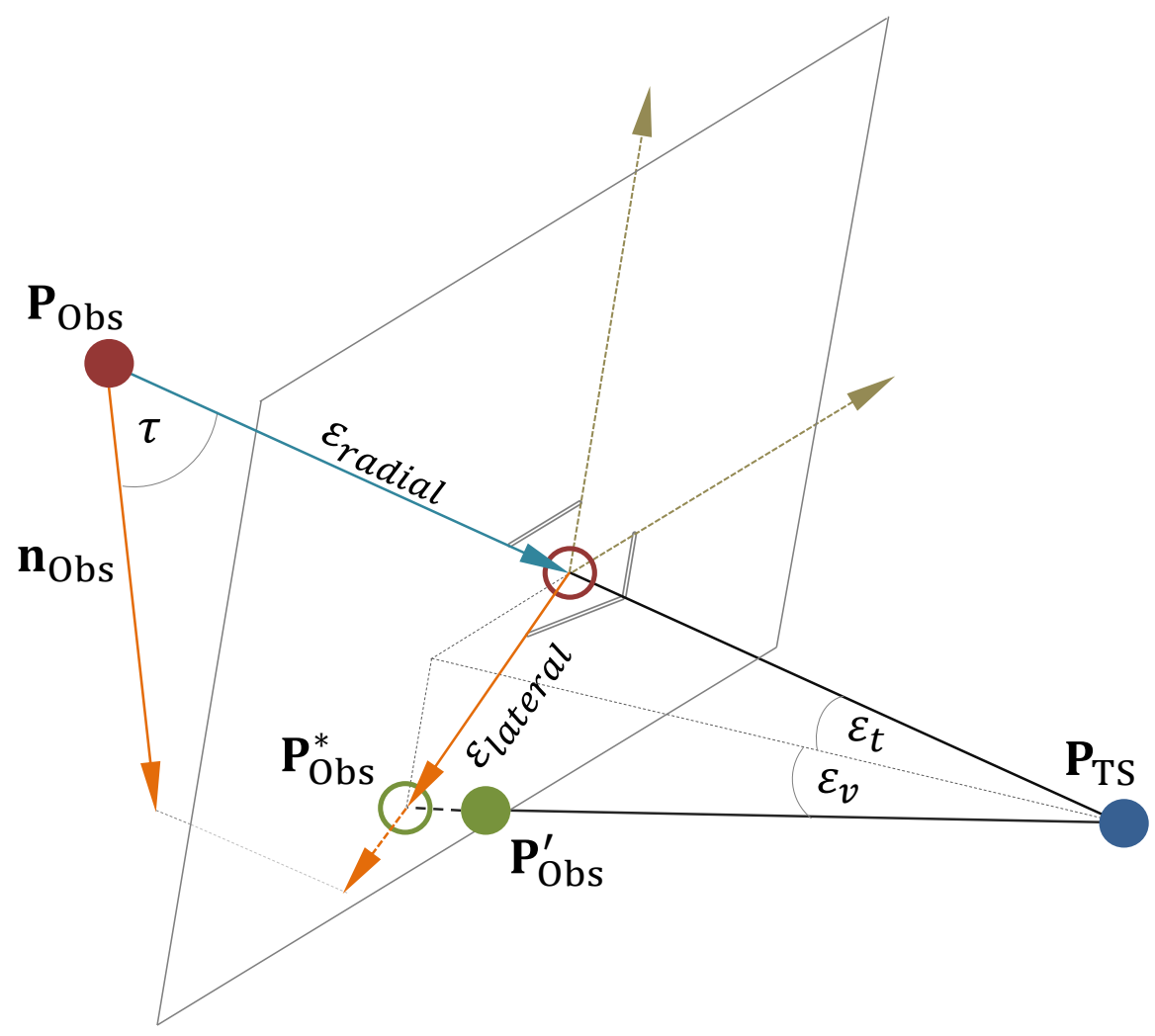

Fig. 4 Schematic representation of the necessary corrections to be applied to the observations due to the target incidence angle at glass prisms, i.e. the misalignment of the normal vector of a glass prism with respect to the survey instrument. The true position of the glass prism is denoted by $\mathrm{P}_{\text {Obs }}^{\prime}$, but due to the properties of the glass prism the survey instrument measures direction and distance towards point $\mathrm{P}_{\mathrm{Obs}}$. Radial and lateral errors $\varepsilon_{\text {radial }}$ and $\varepsilon_{\text {lateral }}$, respectively, are caused. Corresponding corrections $\varepsilon_{\text {radial }}$ for the distance measurement and $\varepsilon_{\mathrm{v}}$ and $\varepsilon_{\mathrm{t}}$ for the vertical and horizontal angle, respectively, have to be applied. See text for further details.

and 8 . The distance measurement can then be corrected directly by $\varepsilon_{\text {radial }}$ while the lateral component $\varepsilon_{\text {lateral }}$ needs to be split-up into a horizontal and a vertical component. To do so, $\mathbf{n}_{\text {Obs }}^{i}$, the normal vector of the survey prism, is projected into the observation plane, giving the projection vector $\mathbf{q}_{\text {Obs }}^{i}$ with

$$
\mathbf{q}_{\mathrm{Obs}}^{i}=\mathbf{R}_{\alpha, \epsilon} \mathbf{n}_{\mathrm{Obs}}^{i}-\left(\frac{\left[\mathbf{P}_{\mathrm{Obs}}^{i}-\mathbf{P}_{\mathrm{TS}}\right]^{T} \mathbf{R}_{\alpha, \epsilon} \mathbf{n}_{\mathrm{Obs}}^{i}}{\left[\mathbf{P}_{\mathrm{Obs}}^{i}-\mathbf{P}_{\mathrm{TS}}\right]^{T}\left[\mathbf{P}_{\mathrm{Obs}}^{i}-\mathbf{P}_{\mathrm{TS}}\right]}\right)\left[\mathbf{P}_{\mathrm{Obs}}^{i}-\mathbf{P}_{\mathrm{TS}}\right]
$$

Using this projection vector a point $\mathbf{P}_{\text {Obs }}^{*}$ in the direction of the true position $\mathbf{P}_{\text {Obs }}^{\prime}$ can be determined:

$$
\mathbf{P}_{\text {Obs }}^{*}=\mathbf{P}_{\text {Obs }}^{i}-\frac{\left|\varepsilon_{\text {lateral }}\right|}{\left|\mathbf{q}_{\text {Obs }}^{i}\right|} \mathbf{q}_{\text {Obs }}^{i}
$$


The vertical and horizontal angles $v^{*}$ and $t^{*}$ with respect to the point $\mathbf{P}_{\text {Obs }}^{*}$ are the same as for $\mathbf{P}_{\text {Obs }}^{\prime}$. The differences of the vertical and horizontal angles with respect to $\mathbf{P}_{\text {Obs }}$ and $\mathbf{P}_{\text {Obs }}^{*}$, respectively, express the necessary corrections $\varepsilon_{\mathrm{v}}=v^{*}-v$ and $\varepsilon_{\mathrm{t}}=t^{*}-t$ that have to be applied to the originally recorded angles $v$ and $t$. We like to point out here that the calculation of these necessary corrections can be done with sufficient accuracy based on the a priori knowledge of the prism normal vector, survey stand point, telescope azimuth and elevation etc. Thus, the corrections can be calculated and applied in real-time by HEIMDALL during the ongoing monitoring measurements.

In general, the stochastic model for each observation type should be parameterized by at least a constant uncertainty $\sigma_{c}$ and a distance dependent uncertainty $\sigma_{v}$. In particular, the common formulas for the uncertainty of a slope distance, a horizontal direction and a vertical angle measurement, $\sigma_{\mathrm{SD}}$, $\sigma_{\mathrm{HD}}$ and $\sigma_{\mathrm{VA}}$, respectively, are (Lösler, 2012)

$$
\begin{gathered}
\sigma_{\mathrm{SD}}=\sqrt{\sigma_{c, s}^{2}+\left(s \cdot \sigma_{v, s}\right)^{2}} \\
\sigma_{\mathrm{HD} / \mathrm{VA}}=\sqrt{\sigma_{c, a}^{2}+\left(\rho \cdot \frac{\sigma_{v, a}}{s}\right)^{2}}
\end{gathered}
$$

where $s$ denotes the distance and $\rho$ is the conversion factor between radian and gon.

To minimize the impact of inconsistent markers, a free network adjustment needs to be performed. To solve the resulting rank deficiency, four additional restrictions have to be introduced to fix the network translations and the rotation. The derived variance-covariance-matrix $\mathbf{Q}_{\mathrm{xyz}}$ depends only on the observations and represents the network intrinsic uncertainty (Illner, 1985; Koch, 1999; Kotsakis, 2012).

\subsection{Reference point determination module}

To observe targets regardless of the telescope orientation, the transformation model by Lösler (2009) can be used. The model describes a transformation of the point $\mathbf{P}$ between the ground-fixed reference system and the radio telescope system.

$$
\mathbf{P}_{\mathrm{Obs}}=\mathbf{P}_{\mathrm{RP}}+\mathbf{R}_{\theta}^{\mathrm{x}} \mathbf{R}_{\phi}^{\mathbf{y}} \mathbf{R}_{\alpha-\mathbf{O}_{\alpha}}^{\mathbf{z}} \mathbf{R}_{\psi}^{\mathbf{y}}\left(\mathbf{E}_{\mathbf{c c}}+\mathbf{R}_{\epsilon-\mathbf{O}_{\epsilon}}^{\mathbf{x}} \mathbf{P}_{\mathrm{Tel}}\right)
$$

Here $\mathbf{P}_{\text {Tel }}=[b a 0]^{T}$ is a point in the telescope system parameterized by the radial distances to the telescope axes, $\mathbf{E}_{\mathbf{c c}}$ is the eccentricity offset and the angle $\psi$ describes the non-orthogonality between the azimuth- and elevationaxes. The small inclination angle between the azimuth axis and the vertical axis of the observation system is parameterized by $\theta$ and $\phi$. Because of the unknown position of $\mathbf{P}_{\mathrm{Tel}}$ in the telescope system, the azimuth and elevation angle $\alpha$ and $\epsilon$ can only be used as non-oriented direction. To avoid this limitation two 
associated orientation correction angles $O_{\alpha}$ and $O_{\epsilon}$ are introduced. To estimate the reference point $\mathbf{P}_{\mathrm{RP}}$ and the additional telescope parameters, a general least squares adjustment, also known as Gauß-Helmert model, is suggested. Based on Lenzmann and Lenzmann (2004), the vector of observations $\mathbf{1}$, the vector of random errors $\mathbf{v}$ and the vector of $u$ unknown parameters $\mathbf{x}$ are connected by $n$ condition equations $\Psi(\mathbf{v}, \mathbf{x})=h(\mathbf{l}+\mathbf{v}, \mathbf{x})$.

The corresponding normal equation system is

$$
\left(\begin{array}{c}
\mathbf{k} \\
\mathbf{x}-\mathbf{x}_{\mathbf{0}}
\end{array}\right)=-\left(\begin{array}{cc}
\mathbf{B} \mathbf{Q}_{11} \mathbf{B}^{\mathbf{T}} & \mathbf{A} \\
\mathbf{A}^{\mathbf{T}} & \mathbf{0}
\end{array}\right)^{-1}\left(\begin{array}{c}
-\mathbf{B v}_{\mathbf{0}}+\Psi\left(\mathbf{v}_{\mathbf{0}}, \mathbf{x}_{\mathbf{0}}\right) \\
\mathbf{0}
\end{array}\right)
$$

where $\mathbf{A}=\left.\frac{\partial \Psi}{\partial \mathbf{x}}\right|_{\mathbf{v}_{\mathbf{0}}, \mathbf{x}_{\mathbf{0}}}$ and $\mathbf{B}=\left.\frac{\partial \Psi}{\partial \mathbf{v}}\right|_{\mathbf{v}_{\mathbf{0}}, \mathbf{x}_{\mathbf{0}}}$ denote Jacobi matrices which contain the partial derivatives with respect to the unknown parameters $\mathbf{x}$ and the observations $\mathbf{l}$, respectively. The vectors $\mathbf{v}_{\mathbf{0}}$ and $\mathbf{x}_{\mathbf{0}}$ represent approximation values, $\mathbf{k}$ consists of so-called Laplace multipliers and $\mathbf{Q}_{\mathbf{l l}}=\operatorname{diag}\left(\mathbf{Q}_{\mathrm{xyz}} \mathbf{Q}_{\alpha \epsilon}\right)$ is the variance-covariance-matrix. The vector $\mathbf{w}=-\mathbf{B v}_{\mathbf{0}}+\Psi\left(\mathbf{v}_{\mathbf{0}}, \mathbf{x}_{\mathbf{0}}\right)$ contains the misclosures. The solution vectors $\mathbf{v}=\mathbf{Q}_{11} \mathbf{B}^{\mathbf{T}} \mathbf{k}$ and $\mathbf{x}$ are applied as new approximations and the method is repeated until the system converges (cf. Neitzel (2010)).

In Equation 13 a stable environment during the observation process is assumed. However, analysis of the influence of thermal effects on the telescope structure shows significant deformations (e.g. Haas et al. (1999), Wresnik et al. (2007)). According to the convention by Nothnagel (2009), the height variation can be modelled by

$$
z_{\mathbf{P}_{\mathrm{RP}}}\left(\Delta T_{i}\right)=z_{\mathbf{P}_{\mathrm{RP}}}+\left(\gamma_{S} h_{S}+\gamma_{F} h_{F}\right) \Delta T_{i}
$$

where $h_{S}$ is the height of the reference point with respect to the foundation, $h_{F}$ is the height of the foundation and $\gamma_{S}$ and $\gamma_{F}$ are the thermal expansion coefficients for the antenna and for the foundation, respectively. The true value of $z_{\mathbf{P}_{\mathrm{RP}}}$ is shifted to $z_{\mathbf{P}_{\mathrm{RP}}}\left(\Delta T_{i}\right)$ as a consequence of the temperature difference $\Delta T_{i}=T_{i}-T_{0}$ between the temperature of the telescope structure $T_{i}$ and a reference temperature $T_{0}$. In addition to the telescope height, the parameters $a$ and $b$ are also affected.

$$
\begin{aligned}
& a\left(\Delta T_{i}\right)=a\left(1+\gamma_{S} \Delta T_{i}\right) \\
& b\left(\Delta T_{i}\right)=b\left(1+\gamma_{S} \Delta T_{i}\right)
\end{aligned}
$$

To avoid systematic errors caused by thermal expansion, Equation 13 is combined together with equations $15-17$. This means that not the average temperature is used for correcting the reference point height, but each observed target position is corrected by their individual thermal expansion value. Thus, a full spatial compensation is introduced. Equation 15 can be neglected if a direct registration of height variations is possible. Moreover, additional restrictions can be implied during the adjustment process, if specific parameters like 
the eccentricity offset are known with higher accuracy. Applying restrictions, Equation 14 becomes

$$
\left(\begin{array}{ccc}
\mathrm{BQ}_{11} \mathbf{B}^{\mathrm{T}} & \mathbf{A} & \mathbf{0} \\
\mathbf{A}^{\mathrm{T}} & \mathbf{0} & \mathbf{G}^{\mathrm{T}} \\
\mathbf{0} & \mathbf{G} & \mathbf{0}
\end{array}\right)\left(\begin{array}{c}
\mathrm{k}_{1} \\
\mathrm{x}-\mathrm{x}_{0} \\
\mathrm{k}_{2}
\end{array}\right)=\left(\begin{array}{c}
-\mathrm{w} \\
\mathbf{0} \\
-\mathrm{g}
\end{array}\right)
$$

where $\mathbf{G}$ denotes the matrix of restriction and $\mathbf{g}$ is the vector of contradictions (e.g. Kupferer (2005)).

3.5 Combination and time series analysis module

For the analysis of VLBI data it is important to have information on the telescope reference point and the eccentricity offset and their potential variations over time. With just one single local survey the results have to be considered as invariant until a new measurement is carried out. A more advanced analysis becomes possible with more frequent reference point determinations, and in the optimum case continual automated monitoring. With $m$ reference point determinations a recursive parameter estimation can be introduced to combine the $m$ results $\mathbf{x}_{j}$ and the corresponding variance-covariance-matrix $\mathbf{Q}_{\mathbf{x}_{j} \mathbf{x}_{j}}$ of each solution.

$$
\mathbf{x}=\left(\begin{array}{c}
\mathbf{x}_{1} \\
\mathbf{x}_{2} \\
\ldots \\
\mathbf{x}_{m}
\end{array}\right) \quad ; \quad \mathbf{Q}_{\mathbf{x x}}=\left(\begin{array}{cccc}
\mathbf{Q}_{\mathbf{x}_{1} \mathbf{x}_{1}} & \mathbf{0} & \ldots & \mathbf{0} \\
\mathbf{0} & \mathbf{Q}_{\mathbf{x}_{2} \mathbf{x}_{2}} & \mathbf{0} \\
\vdots & & \ddots & \vdots \\
\mathbf{0} & \ldots & \mathbf{0} & \mathbf{Q}_{\mathbf{x}_{m} \mathbf{x}_{m}}
\end{array}\right)
$$

Since each single solution $\mathbf{x}_{j}$ is considered as an independent realisation of a random experiment, the combined solution $\hat{\mathbf{x}}_{j}$ is given by (e.g. Koch (2007))

$$
\hat{\mathbf{x}}_{j}=\hat{\mathbf{x}}_{j-1}+\mathbf{K}_{j-1, j}\left(\mathbf{x}_{j}-\hat{\mathbf{x}}_{j-1}\right)
$$

with the gain matrix

$$
\mathbf{K}_{j-1, j}=\mathbf{Q}_{\hat{\mathbf{x}}_{j-1} \hat{\mathbf{x}}_{j-1}}\left(\mathbf{Q}_{\mathbf{x}_{j} \mathbf{x}_{j}}+\mathbf{Q}_{\hat{\mathbf{x}}_{j-1} \hat{\mathbf{x}}_{j-1}}\right)^{-1}
$$

The variance-covariance-matrix $\mathbf{Q}_{\hat{\mathbf{x}}_{j} \hat{\mathbf{x}}_{j}}$ follows with Koch (2007).

$$
\mathbf{Q}_{\hat{\mathbf{x}}_{j} \hat{\mathbf{x}}_{j}}=\mathbf{Q}_{\hat{\mathbf{x}}_{j-1} \hat{\mathbf{x}}_{j-1}}-\mathbf{K}_{j-1, j} \mathbf{Q}_{\hat{\mathbf{x}}_{j-1} \hat{\mathbf{x}}_{j-1}}
$$

Equation 19 disregards the timeliness of the data though it is expected that the duration of validity is limited with time. Introducing a variance matrix of the process noise $\mathbf{C}_{\mathrm{nn}}=\operatorname{diag}\left(\dot{\sigma}_{x_{\mathbf{P}_{\mathrm{RP}}}}^{2} \dot{\sigma}_{y_{\mathbf{P}_{\mathrm{RP}}}}^{2} \dot{\sigma}_{z_{\mathbf{P}_{\mathrm{RP}}}}^{2}\right)$ relating to the reference point $\mathbf{P}_{\mathrm{RP}}$ the modified variance-covariance-matrix $\mathbf{Q}_{\mathbf{x}_{j} \mathbf{x}_{j}}^{\Delta t}$ is given by (cf. Knickmeyer et al. (1996))

$$
\mathbf{Q}_{\mathbf{x}_{j} \mathbf{x}_{j}}^{\Delta t}=\mathbf{Q}_{\mathbf{x}_{j} \mathbf{x}_{j}}+\mathbf{B C}_{\mathrm{nn}} \mathbf{B}^{\mathrm{T}}
$$

where $\mathbf{B}$ is a transfer matrix with respect to the time difference $\Delta t=t_{j}-t_{j-1}$ between the measurements. Substituting $\mathbf{Q}_{\mathbf{x}_{j} \mathbf{x}_{j}}$ by $\mathbf{Q}_{\mathbf{x}_{j} \mathbf{x}_{j}}^{\Delta t}$ in Equation 19 leads to an advanced model. 


\section{The 2012 monitoring campaign at the Onsala Space Observatory}

In June 2012, five monitoring experiments were carried out at the $20 \mathrm{~m}$ VLBI radio telescope at the Onsala Space Observatory. The goal was to use the HEIMDALL monitoring system to perform an automated reference point determination following the previously described concept and to gain experience with this novel approach.

The Onsala Space Observatory is an active network station within the IVS and has a long VLBI history going back to the late 1960s. Since 1979 the $20 \mathrm{~m}$ radio telescope at Onsala is used regularly for geodetic VLBI measurements, and currently about 40 geodetic VLBI sessions are observed every year. The $20 \mathrm{~m}$ radio telescope is enclosed by a protecting radome with a diameter of $30 \mathrm{~m}$. The local survey network inside the radome building consists of five survey pillars on the radome foundation and three ground markers. Figure 5 depicts a cross section and top view of the radome.

A Leica TS30 total station was used for this measurement campaign and was mounted on the survey pillars. Four Leica standard prisms (GPR121) and six Leica mini prisms (GMP104) were mounted at the radio telescope elevation cabin and at the telescope counterweight. As an example, Figure 6 depicts the left side of the telescope elevation cabin and the survey prisms that were mounted. To reduce instrumental errors of the total station, all observations were carried out in two faces, except for the monitoring during observing plans following VLBI schedules. The latter were carried out with one face measurements only, since the radio telescope during these experiments moved all the time and made measurements in two faces impossible. However, in
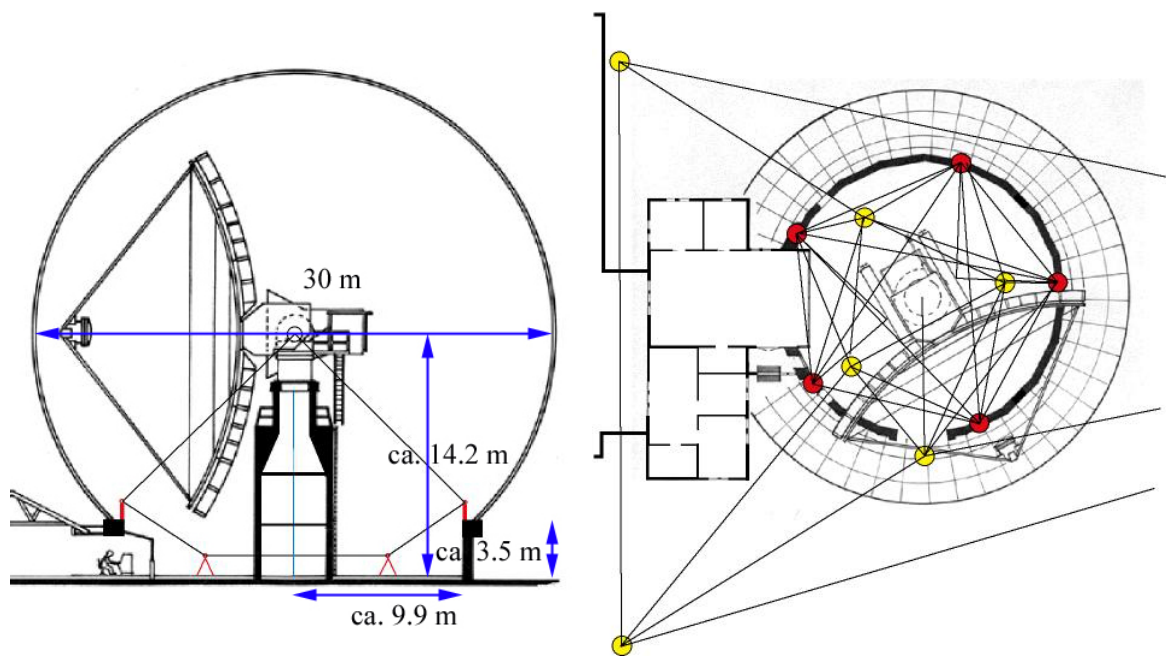

Fig. 5 Left: cross section through the radome enclosed $20 \mathrm{~m}$ radio telescope at the Onsala Space Observatory. Right: top view of the local survey network that consists of survey pillars on the radome foundation (red dots) and survey markers in the ground (yellow dots). 


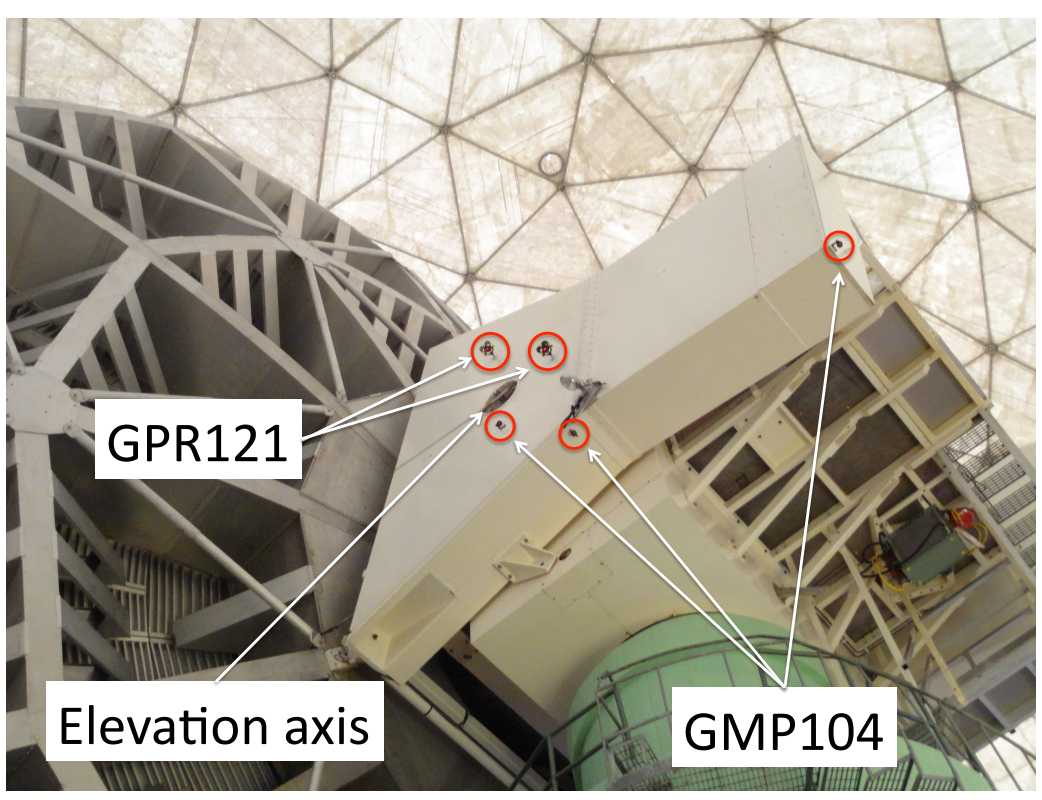

Fig. 6 Left side of the telescope cabin. The red circles indicate where the five survey prisms were attached. Two Leica GPR121 prisms were attached with magnetic supports above and behind the elevation axis, and three Leica GMP104 prisms were attached mechanically on the side of the elevation cabin, below the elevation axis, below and at the end of the counter weight. In a similar way, prisms were attached on the right side of the elevation cabin.

order to minimize instrumental errors also for the one face observations we performed repeatedly the calibration procedures that the manufacturer of the total station advises. These calibration procedures are meant to derive correction values for instrumental errors that then are applied to the measurements to compensate fully for instrumental errors.

The five different monitoring experiments followed four different monitoring approaches. Figure 7 gives an overview of the network configuration of the five experiments. The four monitoring approaches were:

1. DMR: The observation plan was derived based on a dedicated survey schedule (D) (stop-and-go-mode), the observations were performed from multiple survey stand points $(\mathbf{M})$, and the survey targets were observed redundantly (R) and in two faces (see Fig. 7a).

2. DMO: The observation plan was derived based on a dedicated survey schedule (D) (stop-and-go-mode), the observations were performed from multiple survey stand points $(\mathbf{M})$, and each survey target was observed once $(\mathbf{O})$ in two faces (see Fig. 7b).

3. VMO: The observation plan was derived based on a real VLBI schedule $(\mathbf{V})$, the observations were performed from multiple survey stand points $(\mathbf{M})$, and each survey target was observed once $(\mathbf{O})$ in one face only (see Fig. 7c). 
4. VSO: The observation plan was derived based on a real VLBI schedule $(\mathbf{V})$, the observations were performed from a single survey stand point $(\mathbf{S})$, and each survey target was observed once $(\mathbf{O})$ in one face only (see Fig. $7 d$ ).

Considering the homogeneous point cloud for the DMO-approach (see Fig. 7b), we assumed that this approach would provide the best results, and therefore this setup was carried out twice.

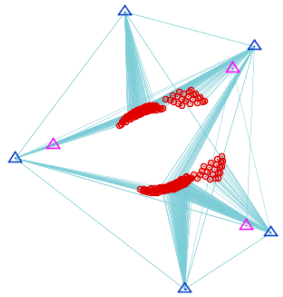

a) DMR

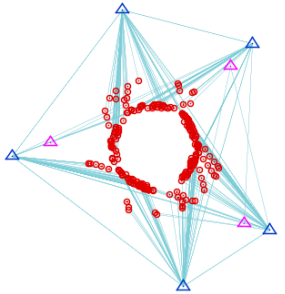

b) $\operatorname{DMO}(2 \mathrm{x})$

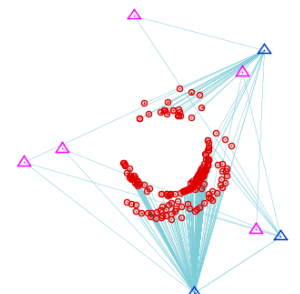

c) $\mathrm{VMO}$

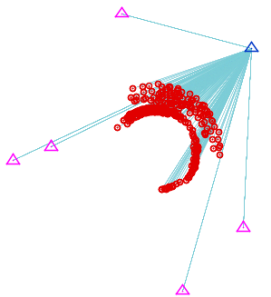

d) VSO

Fig. 7 Horizontal network geometry for the five experiments. Shown are observed connections between the survey pillars of the local survey network (triangles), that define the geodetic datum, and the telescope points (red dots) in the local coordinate system. Blue and magenta triangles depict survey stand points and survey pillar targets, respectively.

A priori measurement uncertainties are needed for the network analysis. These were derived for both, the measurements to the survey targets on the pillars in the local survey network and to the survey targets on the telescope, as described previously in Section 3.3, and are presented in Table 1. While the uncertainties for the measurements to the survey pillars can be derived reliably from a variance-covariance-estimation due to the large measurement redundancy, this is not possible for the targets mounted on the telescope, since those were only observed once (or twice for the DMR approach). Here instead a priori measurement uncertainties were used that are based on the manufacturer's specification for the particular measurement mode of the total station. To accomodate for potential further uncertainties, the a priori measurement uncertainties for the distance measurements were set even larger than the manufacturer's specifications. For distance measurements to telescope targets

Table 1 Stochastic model used for the network adjustment. Presented are the constant uncertainties $\sigma_{\mathrm{c}}$ and distance dependent uncertainties $\sigma_{\mathrm{v}}$ for horizontal directions (HD), vertical angles (VA) and slope distances (SD), for both survey targets mounted on pillars in the local survey network and survey targets mounted on the telescope.

\begin{tabular}{lllllll}
\hline & \multicolumn{2}{c}{ survey pillar targets } & \multicolumn{2}{c}{ telescope targets } & \multicolumn{2}{c}{ telescope targets } \\
& & & \multicolumn{2}{c}{ (for DMO \& DMR) } & \multicolumn{2}{c}{ (for VMO \& VSO) } \\
& $\sigma_{\mathrm{c}}$ & $\sigma_{\mathrm{v}}$ & $\sigma_{\mathrm{c}}$ & $\sigma_{\mathrm{v}}$ & $\sigma_{\mathrm{c}}$ & $\sigma_{\mathrm{v}}$ \\
\hline HD & $0.15 \mathrm{mgon}$ & $0.3 \mathrm{~mm}$ & $0.15 \mathrm{mgon}$ & $3.0 \mathrm{~mm}$ & $0.15 \mathrm{mgon}$ & $3.0 \mathrm{~mm}$ \\
$\mathrm{VA}$ & $0.15 \mathrm{mgon}$ & $0.4 \mathrm{~mm}$ & $0.15 \mathrm{mgon}$ & $3.0 \mathrm{~mm}$ & $0.15 \mathrm{mgon}$ & $3.0 \mathrm{~mm}$ \\
$\mathrm{SD}$ & $0.3 \mathrm{~mm}$ & $1.0 \mathrm{ppm}$ & $1.0 \mathrm{~mm}$ & $1.0 \mathrm{ppm}$ & $3.0 \mathrm{~mm}$ & $1.0 \mathrm{ppm}$ \\
\hline
\end{tabular}


during dedicated schedules an a priori measurement uncertainty of $1 \mathrm{~mm}$ was used. For the measurements in tracking mode during VLBI-schedules we used the manufacturer's measurement uncertainty of $3 \mathrm{~mm}$. Regarding the slow telescope motion to compensate for earth rotation, this uncertainty level is rather pessimistic assumption.

For the restricted measurement volume of our surveys the uncertainty of the distance measurement is strongly dependent on the constant part in Equation 11, while the uncertainty of the angle measurements depends mainly on the distance dependent part in Equation 12. Thus, the main influence of the angle uncertainty is caused by the centering error of the ATR and the residual error of the applied prism correction (see Section 3.3).

The geodetic datum is defined by the local site network (cf. Figure 5). The variance-covariance-matrix $\mathbf{Q}_{\mathrm{xyz}}$ of the network adjustment is required in order to prepare the stochastic model in Equation 14 for the reference point determination. Furthermore, the azimuth and elevation angles were considered to be uncorrelated and having equal uncertainties, so that $\mathbf{Q}_{\alpha \epsilon}$ had a diagonal structure. For a dedicated survey schedule where the telescope operates in stop-and-go mode the angular uncertainties were set to $\sigma_{\alpha}=\sigma_{\epsilon}=0.0015^{\circ}$. To reflect the uncertainties of the timing synchronization between the telescope angles and the total station observations during a VLBI schedule, a less restrictive stochastic model was used. Assuming that the timing synchronization error is less than $2 \mathrm{~s}$, the worst case angular uncertainties become $\sigma_{\alpha}=\sigma_{\epsilon}=0.01^{\circ}$.

In general, each telescope point is only observed once and outliers can not be identified using a statistical test during the network adjustment. Lösler (2009) suggests several multiple stochastic tests to check the introduced target positions and telescope angles during the reference point determination. The tested observation with a maximum exceeding of a defined threshold was excluded. This procedure was repeated until no more outlier was detected.

Table 2 summarizes the results of the five experiments. The presented uncertainties are the formal errors that were multiplied by a scaling factor in order to give realistic values. This scaling factor was estimated individually for the different monitoring experiments and depends both on the ratio of the mean point error and the formal error of the reference point, and on an empirical measure to describe the homogeneity of the measurement setup. The first ingredient of the scaling factor is based on a hypothetic consideration that the reference point could have been observed directly, just like any other survey target on the telescope. A reasonable assumption is therefore that the uncertainty of the reference point cannot be smaller than the point error of an observed survey target. Thus, one ingredient of the scaling factor is the ratio of the mean error of the survey points and the formal error of the reference point. The other ingredient for the scaling factor is an expression for the homogeneity of the measurement setup. It is reasonable to assume that the reference point can be determined more reliably when measurements from all survey pillars around the telescope are performed, i.e. a homogenous point cloud can be achieved. In this case the reference point is enclosed within a point cloud and 
a situation is avoided that the reference point is located behind the point cloud, which would correspond to some kind of extrapolation. Also potential wobble errors of the telescope will be compensate with a homogenous point cloud. We thus introduce a measure to describe the degree of homogeneity. It can be approximated for our case by the ratio of number of possible survey stand points, $d_{\max }$ with respect to the number of used survey stand points, $d$. Using this definition, solutions S2 and S4 that use five out of five possible survey stand points have a more ideal network configuration than the other solutions (see Figure 7). Following the above described argumentation the estimated uncertainties of the parameters were scaled-up by the factor $\mu$, with

$$
\mu=\frac{\frac{1}{k} \sum_{i=1}^{k} \sqrt{\operatorname{tr}\left(\mathbf{Q}_{i i}\right)}}{\sqrt{\operatorname{tr}\left(\mathbf{Q}_{\left.\mathbf{P}_{\mathrm{RP}}\right)}\right.}} \cdot \frac{d_{\max }}{d}
$$

Here the total number of survey points included in the adjustment process is $k$ and $\hat{\sigma} \sqrt{\operatorname{tr}(\mathbf{Q})}$ describes the estimated error for the spatial position of a point (Ghilani and Wolf, 2006). The scaling factors for the five monitoring experiments are given in Table 2, too. Figure 8 presents the results for the reference point coordinates and their error bars expressed relative with respect to the first solution, and the absolute results and error bars of the eccentricity.

Comparing the results of the five experiments we see that the results agree rather well with each other. The eccentricity agrees for all five solutions within less than $\pm 0.5 \mathrm{~mm}$. Solutions S1, S2, S3 and S4 agree for the reference point within less than $\pm 0.2 \mathrm{~mm}$ for the X-component, and within less than $\pm 1 \mathrm{~mm}$ for the Y- and Z-components. Solutions S2 and S4 with the most homogeneous measurement situation agree within less than $\pm 0.3 \mathrm{~mm}$ for all four parameters. However, solution S5 deviates from the other four solutions. For S5 the Xcomponent deviates by up to $2.9 \mathrm{~mm}$, the Y-component by up to $3.5 \mathrm{~mm}$, and the Z-component by up to $6.2 \mathrm{~mm}$ with respect to the other solutions. This particular solution was carried out with data observed during a VLBI schedule

Table 2 Reference point coordinates and axis eccentricity derived from the five individual monitoring experiments.

\begin{tabular}{llllll}
\hline Solution & S1 & S2 & S3 & S4 & S5 \\
\hline Approach & DMR & DMO & VMO & DMO & VSO \\
Survey stand points & 5 & 5 & 3 & 5 & 1 \\
Redundancy $(\mathrm{r}=\mathrm{n}-\mathrm{u})$ & 922 & 760 & 592 & 940 & 2134 \\
Scaling factor $\mu$ & 1.0 & 1.6 & 3.1 & 1.7 & 6.9 \\
$x_{\mathbf{P}_{\mathrm{RP}}(\mathrm{m})}$ & 90.1238 & 90.1236 & 90.1236 & 90.1237 & 90.1265 \\
& \pm 0.0004 & \pm 0.0001 & \pm 0.0004 & \pm 0.0001 & \pm 0.0008 \\
$y_{\mathbf{P}_{\mathrm{RP}}}(\mathrm{m})$ & 35.9500 & 35.9492 & 35.9483 & 35.9492 & 35.9518 \\
& \pm 0.0004 & \pm 0.0001 & \pm 0.0005 & \pm 0.0001 & \pm 0.0008 \\
$z_{\mathbf{P}_{\mathrm{RP}}}(\mathrm{m})$ & 22.7591 & 22.7597 & 22.7584 & 22.7602 & 22.7540 \\
& \pm 0.0002 & \pm 0.0004 & \pm 0.0012 & \pm 0.0004 & \pm 0.0016 \\
$E_{\mathrm{CC}}(\mathrm{mm})$ & -5.6 & -6.1 & -6.5 & -6.2 & -6.1 \\
& \pm 0.4 & \pm 0.2 & \pm 1.2 & \pm 0.2 & \pm 0.8 \\
\hline
\end{tabular}


and only from one survey stand point. This means that the measurements were taking in just one face and with a geometry that was far from optimum. The fact that measurements were only done from one survey stand point gives an inhomogeneous measurement situation and explains the deviation from the results obtained from the other solutions. For this solution the reference point is always located behind the point cloud which leads to some kind of extrapolation. The fact that the measurements were just taken in one face is not so important, as can seen from solution S3. Solution S3 also used one face measurements only, but the measurements were performed from three survey stand points, thus providing a much better geometrical situation. The results from solution S3 agree better with the others than the results from S5 do. Although the VLBI schedule concomitant experiments S3 and S5 have some weaknesses, the results confirm the feasibility of the automated monitoring.

In general, except for solution S1, the uncertainties of the vertical position are larger than for the horizontal position. The reason for the smaller uncertainties for the horizontal positions than for vertical positions in solution S1 could not be explained completely and is still under investigation. It does not result from the redundant measurement arrangement, since an analysis without considering multiple measurements, i.e. in the style of DMO, gave similar results. However, comparing the observation geometry of S1 (Fig. 7a) with the one of S2 and S4 (see Fig. 7b) it becomes clear that the telescope has not been rotated over the same azimuth range for S1 than for S2 and S4. The azimuth range for S1 was much smaller than for S2 and S4, and the survey targets were only measured on two opposite sides, but not all around the telescope. This could explain the deviations seen between the results of S1 and those of S2 and S4.

Following the approach described in Section 3.5, 'smoothed' results were derived by combining the five individual solutions. These smoothed results for the reference point coordinates and the eccentricity offset are presented in Table 3. Each solution affects the estimated parameters in an attenuated way. The influence of each solution can be controlled by $\mathbf{C}_{\mathrm{nn}}$ (see Section 3.5). Considering the larger uncertainty of $z_{\mathrm{P}_{\mathrm{RP}}}$ and the stable situation at On-

Table 3 'Smoothed' results from combining successive measurement campaigns. Shown are results for the reference point coordinates $x_{\mathbf{P}_{\mathrm{RP}}}, y_{\mathbf{P}_{\mathrm{RP}}}, z_{\mathbf{P}_{\mathrm{RP}}}$ and the eccentricity $E_{\mathrm{CC}}$. Combinations $\mathrm{C} 1, \mathrm{C} 2, \mathrm{C} 3$ and $\mathrm{C} 4$ show the combinations of solutions $\mathrm{S} 1+\mathrm{S} 2, \mathrm{~S} 1+\mathrm{S} 2+\mathrm{S} 3$, $\mathrm{S} 1+\mathrm{S} 2+\mathrm{S} 3+\mathrm{S} 4$, and $\mathrm{S} 1+\mathrm{S} 2+\mathrm{S} 3+\mathrm{S} 4+\mathrm{S} 5$, respectively.

\begin{tabular}{lllll}
\hline & $\mathrm{C} 1$ & $\mathrm{C} 2$ & $\mathrm{C} 3$ & $\mathrm{C} 4$ \\
\hline$x_{\mathbf{P}_{\mathrm{RP}}}[\mathrm{m}]$ & 90.1236 & 90.1236 & 90.1236 & 90.1236 \\
& \pm 0.0002 & \pm 0.0001 & \pm 0.0001 & \pm 0.0001 \\
$y_{\mathbf{P}_{\mathrm{RP}}[\mathrm{m}]}$ & 35.9493 & 35.9492 & 35.9492 & 35.9492 \\
& \pm 0.0002 & \pm 0.0002 & \pm 0.0001 & \pm 0.0001 \\
$z_{\mathbf{P}_{\mathrm{RP}}[\mathrm{m}]}$ & 22.7592 & 22.7592 & 22.7593 & 22.7592 \\
& \pm 0.0002 & \pm 0.0002 & \pm 0.0002 & \pm 0.0002 \\
$E_{\mathrm{CC}}[\mathrm{mm}]$ & -6.1 & -6.1 & -6.1 & -6.0 \\
& \pm 0.2 & \pm 0.2 & \pm 0.1 & \pm 0.1 \\
\hline
\end{tabular}


sala (Lösler and Haas, 2009) a recursive adjustment was carried out choosing $\dot{\sigma}_{x_{\mathbf{P}_{\mathrm{RP}}}}=\dot{\sigma}_{y_{\mathbf{P}_{\mathrm{RP}}}}=0.001 \mathrm{~m} / \mathrm{a}$ and $\dot{\sigma}_{z_{\mathbf{P}_{\mathrm{RP}}}}=0.003 \mathrm{~m} / \mathrm{a}$, and therefore $\mathbf{B C}_{\mathrm{nn}} \mathbf{B}^{\mathrm{T}}=\Delta t^{2} \cdot \operatorname{diag}\left(\dot{\sigma}_{x_{\mathrm{P}_{\mathrm{RP}}}}^{2} \dot{\sigma}_{y_{\mathrm{P}_{\mathrm{RP}}}}^{2} \dot{\sigma}_{z_{\mathrm{P}_{\mathrm{RP}}}}^{2} 0\right)$. Figure 8 shows both the results derived from the five individual solutions (blue points with 3- $\sigma$ error bars) and the smoothed results based on the filter approach (green line with $3-\sigma$ error bounds).

A standard epoch-based determination of a reference point only uses measurements of one particular survey epoch and ignores prior information. In contrast to this allows the recursive parameter estimation to involve all prior information and the corresponding variance-covariance-matrices. The estimation process is therefore based on a larger data set than just one epoch and thus reliable results can be derived. Regardless of the uncertainties of a single solution, the uncertainties of the combined results reduce, if the results of the single solutions are aligned. This becomes clearly visible in Figure 8 for the combination of the first four epochs. The impact of the fifth epoch on the
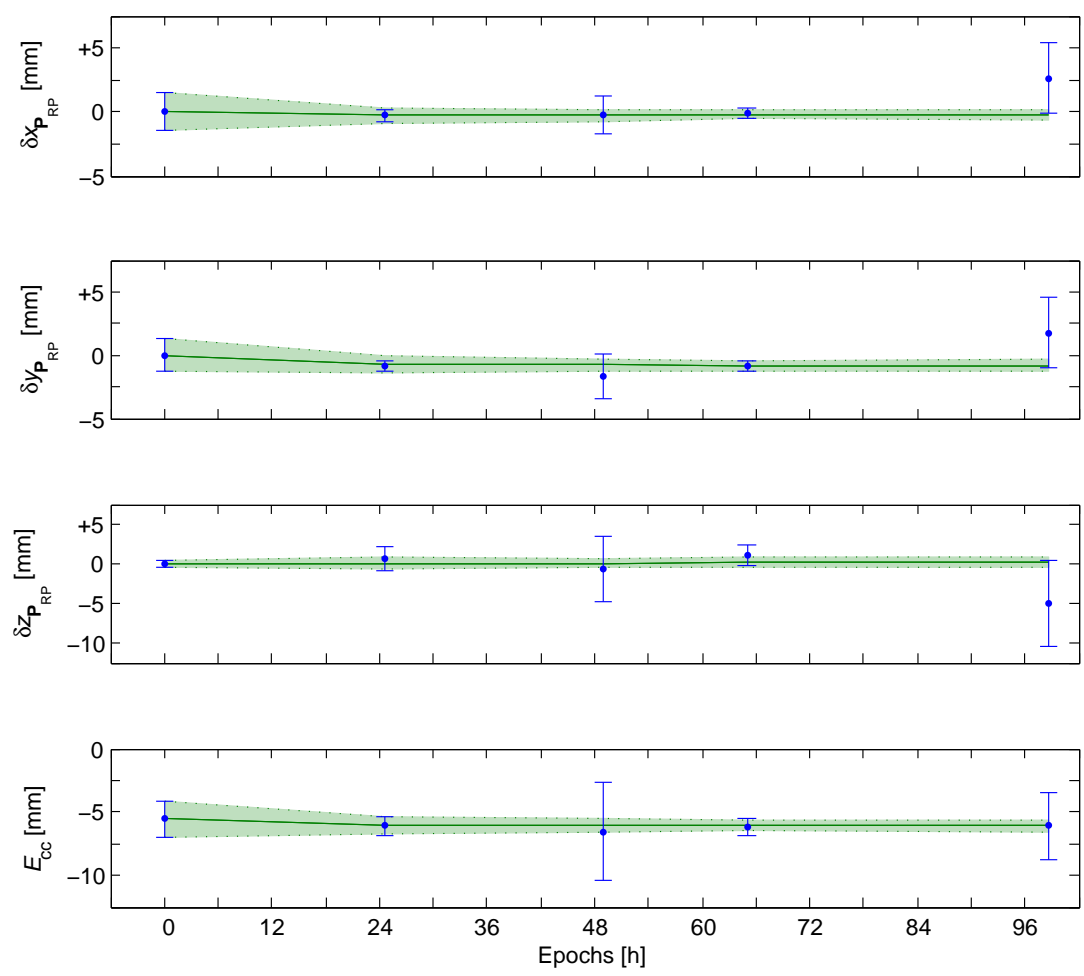

Fig. 8 Top three panels: Time series of the variations of the reference point coordinates $\left(x_{\mathbf{P}_{\mathrm{RP}}}, y_{\mathbf{P}_{\mathrm{RP}}}, z_{\mathbf{P}_{\mathrm{RP}}}\right)$ with respect to the first epoch. Bottom panel: Time series of the telescope axis offset $\left(E_{\mathrm{cc}}\right)$. The blue dots with $3-\sigma$ error bars are the individual determinations based on the five experiments. The smoothed results are shown as green line with a $3-\sigma$ error range. 
combined results is rather small, although the results of this epoch deviate significantly from the other epochs. Due to its memory function, the recursive parameter estimation allows to combine also less favorable observation configurations, e.g. VSO, that themselves would not fulfill the accuracy requirements. The recursive parameter estimation thus increases the robustness of the solution and provides sub-mm accuracy.

Since the five experiments were carried out during 5 consecutive days, any attempt to perform a time series analysis is still too early. To derive further conclusions a longer time series is necessary. In general, reliable results can be expected, if the allocation of the observed targets are homogeneous.

\section{Summary and conclusions}

Within the framework of GGOS, automated and continual determinations with sub-mm accuracy of the reference points of space geodetic instruments are discussed. We developed a monitoring system that answers to this request and tested and evaluated it during a measurement campaign at the Onsala Space Observatory in the summer of 2012. The monitoring system is called HEIMDALL and uses Java-based sensor communication and SQL database management. It involves several modules, including the preparation of an observing plan, the automated monitoring, the network adjustment, the reference point determination, and the combination of solutions. To our knowledge HEIMDALL is the first survey system that allows automated and continual monitoring of the reference point of a radio telescope even during normal operation of the telescope.

The reference point of the $20 \mathrm{~m}$ radio telescope at Onsala was determined with this monitoring system during five experiments following four different approaches. The telescope reference point and the eccentricity offset were estimated from several non-predefined target positions, using the transformation model by Lösler (2009) with a rigorous Gauß-Helmert model. The formal errors of the results were scaled up to provide reasonable uncertainties. This scaling was done with individual experiment-dependent scaling factors that take into consideration both the mean formal error of a survey target on the telescope and the measurement geometry.

The first four experiments involving several survey stand points and generating homogenous point clouds provide results that are in very good agreement with each other and are to be preferred from approaches leading to inhomogeneous point clouds. The fifth experiment was performed during a VLBI schedule and used only one survey stand point leading to an inhomogeneous point cloud, and the corresponding results show deviations on the order of several $\mathrm{mm}$ with respect to the other solutions. A strategy to achieve homogeneous point clouds also during VLBI schedules could be to move the total station every 8-12 hours to a different survey pillar. Disregarding this fifth experiment where only one survey stand point was used, the results for the reference point coordinates and the eccentricity offset agree within $\pm 1 \mathrm{~mm}$ 
or better. The results from the two experiments with the most homogeneous measurement geometry agree even within less than $\pm 0.3 \mathrm{~mm}$ for all parameters. This can be regarded as a proof that not only sub-mm precision but also sub-mm accuracy can be achieved with HEIMDALL.

HEIMDALL includes also a method to combine the results from several experiments using a recursive estimation procedure. For each new determination of the telescope parameters this recursive approach makes use of a priori information in terms of previous results. It thus makes use of continual measurements in a very suitable way, exploiting the information content of the individual experiments. This approach allows even to incorporate experiments that suffered from a less favorable measurement geometry. Applying this approach to our experiments sub-mm accuracies for the reference point coordinates and the eccentricity offset were achieved. The experience so far is limited to measurements performed just during a couple of days. Longer time series are needed to improve the combination procedure.

We explicitly addressed the consequences of observing standard glass body prisms on a rotating structure, like a radio telescope, when the survey target cannot be aligned with respect to the survey instrument. Systematic deviations in the estimated positions of the survey targets are caused that depend on the angle of incidence with respect to the normal on the target glass body. We therefore derived in Section 3.3 corresponding correction formulae that are based on the incident angle in order to be able to correct for these significant errors. The incidence angle can be calculated with sufficient accuracy using Equation 6 to ensure sub-mm accuracy (see Figure 3). To our knowledge this is the first time that this error contribution has been treated correctly in a deterministic way for the determination of a radio telescope reference point.

While we so far have concentrated on the automated and continual determination of the radio telescope reference point, we are now working on the further development of HEIMDALL. The aim is to extend the system to handle complete local tie surveys, again in an automated and continual approach, and will e.g. require the incorporation of additional total stations. We also aim at an improved blunder discovery during the monitoring to reduce the adjustment time.

Acknowledgements The authors thank Anja Mazura and Matthias Burg for supporting this work by their bachelor's theses.

\section{References}

Burg M (2012) Einrichtung des Monitoringsystems HEIMDALL zur automatischen Bestimmung des IVS-Referenzpunktes an der Fundamentalstation Onsala. Bachelor's thesis, University of Applied Sciences Frankfurt am Main, unpublished. 
Crocetto N, Gatti M, Russo P (2000) Simplified formulae for the BIQUE estimation of variance components in disjunctive observation groups. Journal of Geodesy, 74, 447-457.

Ghilani CD, Wolf PR, (2006) Adjustment Computations - Spatial Data Analysis, Wiley and Sons, Hoboken, New Jersey, 4nd edn.

Dawson J, Sarti P, Johnston G, Vittuari L (2007) Indirect approach to invariant point determination for SLR and VLBI systems: an assessment. Journal of Geodesy, 81, 433-441.

Determination of the IVS reference point at the Onsala Space Observatory in a local geodetic Reference frame. Diploma thesis. University of Karlsruhe, unpublished.

Favre C, Hennes M (2000) Zum Einfluss der geometrischen Ausrichtung von 360-Reflektoren bei Messungen mit automatischer Zielerfassung. Vermessung, Photogrammetrie, Kulturtechnik, 2, 72-78.

Haas R, Nothnagel A, Schuh H, Titov O (1999) Explanatory supplement to the section 'Antenna Deformation' of the IERS Conventions (1996). In: DGFI report nr. 71, edited by H. Schuh, Deutsches Geodätisches Forschungsinstitut (DGFI), Munich (Germany), 26-29.

Haas R, Bergstrand S (2010) COLD MAGICS - Continuous Local Deformation Monitoring of an Arctic Geodetic Fundamental Station. In: VLBI2010: From Vision to Reality, IVS 2010 General Meeting Proceedings, NASA/CP-2010215864, edited by D. Behrend and K. D. Baver, 118-122.

Haas R, Bergstrand S, Lehner W (2013) Evaluation of GNSS Monument Stability. In: Reference Frames for Applications in Geosciences, International Association of Geodesy Symposia, 138, edited by Z. Altamimi and X. Collieux, 45-50.

Illner I (1985) Datumsfestlegung in freien Netzen. Dissertationen, Deutsche Geodätische Kommission, Reihe C, 309, München.

Kallio U, Poutanen M (2012) Can We Really Promise a mm-Accuracy for the Local Ties on a Geo-VLBI Antenna. In: Geodesy for Planet Earth, International Association of Geodesy Symposia, 136, edited by S. Kenyon, M. C. Pacino, U. Marti, Springer-Verlag Berlin Heidelberg,doi:10.1007/ 978-3-642-20338-1_5

Knickmeyer ET, Knickmeyer EH, Nitschke M (1996) Zur Auswertung kinematischer Messungen mit dem Kalman-Filter. Schriftenreihe des Deutschen Vereins für Vermessungswesen (DVW), 22, 141-166, Stuttgart.

Koch KR (1999) Parameter Estimation and Hypothesis Testing in Linear Models, Springer, Heidelberg/Berlin, 2nd edn.

Koch KR (2007) Introduction to Bayesian Statistics, Springer, Heidelberg/Berlin, 2nd edn.

Kotsakis C (2012) Reference frame stability and nonlinear distortion in minimum-constrained network adjustment. Journal of Geodesy, 86, 755774 .

Krickel B (2004) Leistungskriterien zur Qualitätskontrolle von Robottachymetern. Dissertationen, Mitteilungen aus den Geodätischen Instituten der Rheinischen Friedrich-Wilhelms-Universität, 92, Bonn. 
Kupferer S (2005) Anwendung der Total-Least-Squares-Technik bei geodätischen Problemstellungen, Dissertationen, Schriftenreihe des Studiengangs Geodäsie und Geoinformatik, Karlsruhe.

Leinen S, Becker M, Dow J, Feltens J, Sauermann K (2007) Geodetic Determination of Radio Telescope Antenna Reference Point and Rotation Axis Parameters, Journal of Surveying Engineering, 133, 41-51.

Lenzmann L, Lenzmann E (2004) Strenge Auswertung des nichtlinearen GaußHelmert-Modells. Allgemeine Vermessungs-Nachrichten, 2, 68-73.

Lösler M (2008) Reference point determination with a new mathematical model at the $20 \mathrm{~m}$ VLBI radio telescope in Wettzell. Journal of Applied Geodesy, 2, 233-238.

Lösler M (2009) New Mathematical Model for Reference Point Determination of an Azimuth-Elevation Type Radio Telescope. Journal of Surveying Engineering, 135, 131-135.

Lösler M, Haas R (2009) The 2008 Local-tie Survey at the Onsala Space Observatory. In: Proceedings of the EVGA - European VLBI for Geodesy and Astrometry, March 23.-24., Bordeaux (France), edited by G. Bourda, P. Charlot and A. Collioud, 97-101.

Lösler M, Eschelbach C, Schenk A, Neidhardt A (2010) Permanentüberwachung des 20m VLBI-Radioteleskops an der Fundamentalstation in Wettzell. Zeitschrift für Geodäsie, Geoinformatik und Landmanagement, 1, 40-48.

Lösler M (2012) JAG3D - Ein kostenfreies Programm zur Netzausgleichung und Deformationsanalyse. In: tech12 - Aktuelle Trends der Messdatenauswertung in Kataster- und Ingenieurvermessung, edited by F. Neitzel.

Mazura A (2012), Erstellung eines Beobachtungsplans für das terrestrische Monitoring von Radioteleskopen. Bachelor's thesis, University of Applied Sciences Frankfurt am Main, unpublished.

Neitzel F (2010) Generalization of total least-squares on example of unweighted and weighted 2D similarity transformation. Journal of Geodesy, 84, 751-762.

Nothnagel A (2009) Conventions on thermal expansion modelling of radio telescopes for geodetic and astrometric VLBI. Journal of Geodesy, 83, 787792.

Pauli W (1969) Vorteile eines kippbaren Reflektors bei der elektrooptischen Streckenmessung. Vermessungstechnik, 17, 412-415.

Plag HP, Pearlman M (Eds.) (2009) The Global Geodetic Observering System. Meeting the Requirements of a Global Society on an Changing Planet in 2020, Springer, Heidelberg/Berlin.

Ray J, Altamimi Z (2005) Evaluation of co-location ties relating the VLBI and GPS reference frames. Journal of Geodesy, 79, 189-195.

Rothacher M, Beutler G, Behrend D, Donnellan A, Hinderer J, Ma C, Noll C, Oberst J, Pearlman M, Plag H-P, Richter B, Schöne T, Tavernier G, Woodworth PL (2009) The future Global Geodetic Observing System. In: H-P Plag and M Pearlman: The Global Geodetic Observering System. Meeting the Requirements of a Global Society on an Changing Planet in 2020, Springer, Heidelberg/Berlin, 2009, doi : 10.1007/978-3-642-02687-4, 237-272. 
Rummel R, Rothacher M, Beutler G (2005) Integrated Global Geodetic Observing System (IGGOS) - science rationale. Journal of Geodynamics, 40, 357-362

Rüeger JM (1990) Electronic Distance Measurement - An Introduction, Springer, Heidelberg/Berlin, 3rd edn.

Sarti P, Sillard P, Vittuari L (2004), Surveying co-located space-geodetic instruments for ITRF computation. Journal of Geodesy, 78, 210-222.

Schmeing S, D. Behrend D, J. Gipson J, Nothnagel A (2010) Proof-of-Concept Studies for a Local Tie Monitoring System. In: VLBI2010: From Vision to Reality, IVS 2010 General Meeting Proceedings, NASA/CP-2010-215864, edited by D. Behrend and K. D. Baver, 360-364.

Wresnik J, Haas R, Böhm J, Schuh H (2007) Modeling thermal deformation of VLBI antennas with a new temperature model. Journal of Geodesy, 81, 423-431. 